$>$ Les mucormycoses sont des infections invasives provoquées par des champignons filamenteux ubiquitaires appartenant l'ordre des Mucorales. Elles surviennent notamment chez des patients immunodéprimés, diabétiques ou ayant subi une transplantation d'organe. À l'heure actuelle, il n'existe pas de recommandations françaises pour le diagnostic de laboratoire des mucormycoses. Ce diagnostic repose principalement sur l'examen histologique et la mise en culture de l'échantillon clinique. II n'existe pas de techniques standardisées qui soient une alternative au diagnostic microbiologique. Nous récapitulons ici les méthodes microbiologiques utilisées dans la démarche diagnostique de la maladie ainsi que dans l'identification des Mucorales. <

\section{Diagnostic microbiologique des mucormycoses}

Dea Garcia-Hermoso

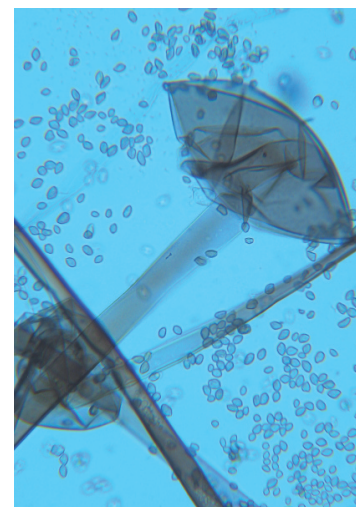

Institut Pasteur, Unité de Mycologie Moléculaire et Centre National de Référence Mycoses Invasives et Antifongiques, CNRS URA3012, 25 , rue du Docteur Roux, 75724 Paris Cedex 15, France. dea.garcia-hermoso@pasteur.fr

capables de contaminer des fruits ou des céréales [9]. Ces champignons sont aussi fréquemment rencontrés comme contaminants de laboratoire. Ils peuvent cependant provoquer des infections nosocomiales [10].

\section{Taxonomie}

La position taxonomique des Mucorales dans la classification du règne Fungi a subi de nombreux changements dus aux récentes études phylogénétiques qui établissent la nature polyphylétique de ce groupe [1113]. Pour cette raison, la division Zygomycota a été abandonnée et les ordres de Mucorales/Mortierellales et Entomophthorales ont été assignés au niveau de sous-divisions Mucoromycotina et Entomophthoromycotina respectivement [14]. De ce fait, les termes Zygomycota ou Zygomycetes n'ont aucune valeur taxonomique. Les Mucorales pathogènes pour l'homme comportent les genres Rhizopus, Lichtheimia, Rhizomucor, Mucor, Cunninghamella, Saksenaea, Cokeromyces, Apophysomyces et Syncephalastrum. Parmi eux, Rhizopus, Lichtheimia, Rhizomucor et Mucor sont les pathogènes les plus fréquemment isolés $[1,2,15,16]$.

\section{Biologie et morphologie des Mucorales}

Ces champignons se reproduisent sexuellement par la formation des zygospores après fusion de deux filaments parfois issus de la même spore (homothallique) et plus souvent de deux filaments complémentaires (hétérothallique). La zygospore arrivée à maturité a souvent une paroi épaisse et passe par une période de dormance avant de germer. La multiplication asexuée est caractérisée par la production d'une à plusieurs sporocystospores (endospores) portées dans des structures fermées en forme de sac nommées sporocystes. Les spores sont disséminées 


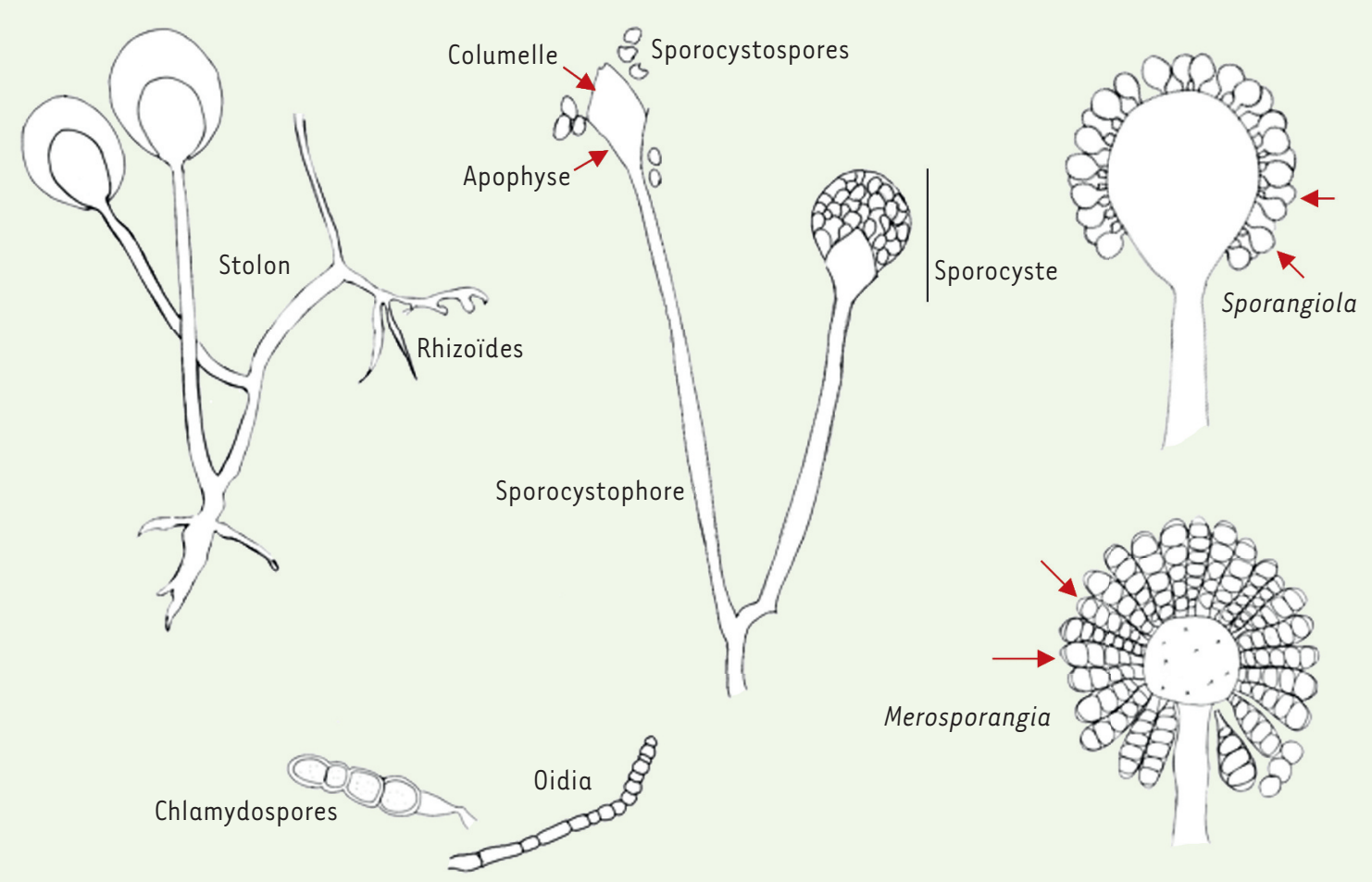

Figure 1. Structures morphologiques des membres de l'ordre des Mucorales.

dans l'atmosphère par éclatement de ces structures. Le sporocyste est supporté par un hyphe spécialisé, sporocystophore, qui, dans certaines espèces, émane d'un système branché de rhizoïdes qui ancrent le sporocystophore au substrat. Les rhizoïdes sont connectés entre eux par un hyphe dénommé stolon, ce qui permet son expansion. D'autres structures sont remarquables tels que l'axe central du sporocyste appelé columelle et l'apophyse, renflement du sporocystophore juste en dessous de la columelle. Le sporocyste peut contenir une ou quelques sporocystospores (sporangiola) ou quelques sporocystospores alignées (merosporangia). Certaines espèces produisent des structures de résistance enflées à paroi épaisse (chlamydospores) ou bien à paroi fine (oidia) [17] (Figure 1).

\section{Diagnostic de laboratoire}

À ce jour, en France, il n'existe pas de recommandation spécifique pour le diagnostic de laboratoire des mucormycoses. L'examen histologique et la mise en culture continuent à être les méthodes de référence pour le diagnostic de cette maladie bien qu'elles ne soient pas considérées comme adaptées à un diagnostic précoce en raison de leur faible sensibilité. Dans certains cas, seule la présence des hyphes caractéristiques constitue l'élément diagnostique de l'infection car les cultures à partir des échantillons tissulaires sont fréquemment négatives. Par ailleurs, dans l'approche histologique, des résultats faussement négatifs ou positifs peuvent être rendus par confusion entre les hyphes des Mucorales et les hyphes déformés de certains hyphomycètes dans les échantillons tissulaires [18]. Les techniques alternatives à la mise en culture basées sur la détection d'antigène (galactomannane, $\beta$-D-glucane) et incluses comme critères diagnostiques des infections fongiques invasives (IFI) [19], n'ont pas d'utilité dans le cas des mucormycoses [20]. De même, nous ne disposons pas à I'heure actuelle d'une technique standardisée et validée pour la détection moléculaire des Mucorales dans les liquides biologiques ou les tissus.

\section{Diagnostic microbiologique à partir des échantillons cliniques}

La démarche diagnostique au laboratoire se base donc sur l'isolement et l'identification des champignons responsables de l'infection. Le choix du site de prélèvement, l'acheminement dans de bonnes conditions pour éviter son desséchement et la prompte manipulation au laboratoire sont des éléments fondamentaux. Divers prélèvements peuvent être envisagés selon les manifestations cliniques et le site d'infection, mais les biopsies tissulaires sont considérées comme les échantillons de choix. L'expectoration, le sang ou le LCR (liquide céphalorachidien) ne sont pas des liquides biologiques utiles pour le diagnostic de mucormycoses. Il est important de noter que les sécrétions respiratoires ou biopsies obtenues devront être d'un volume ou d'une quantité suffisants pour optimiser le rendement [17, 20, 21$]$. 


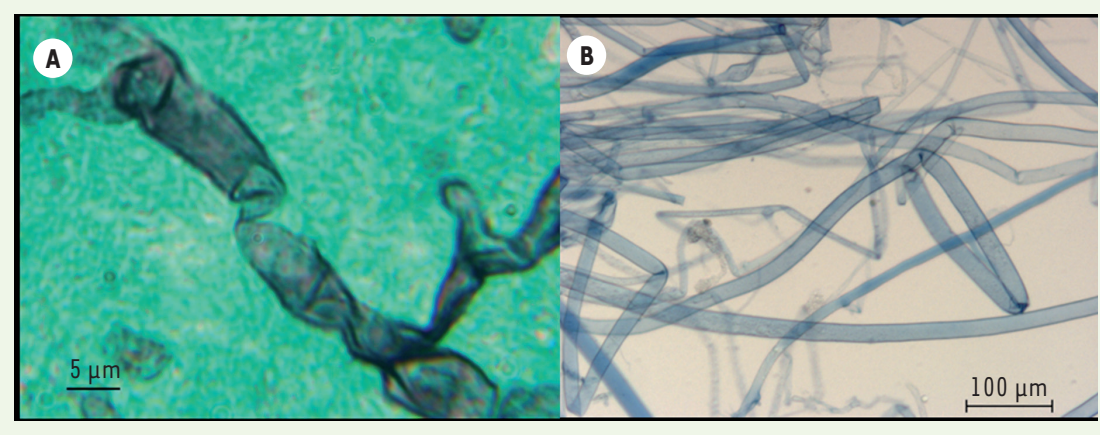

Figure 2. Aspect microscopique des hyphes cœnocytiques $(A)$ après coloration de Gomori-Grocott (B) et par du bleu lactique.

peut provoquer une perte de viabilité des Mucorales liée principalement au caractère cœnocytique et en conséquence fragile du mycélium. II est donc préconisé d'émincer les tissus (sans broyage donc) et plus facile

\section{Examen direct et anatomopathologie}

L'examen direct de tissus frais se fait par observation microscopique après l'ajout de quelques gouttes d'hydroxyde de potassium ( $\mathrm{KOH}$ ). L'utilisation de fluorochromes (calcofluor white, Uvitex2B, Blankophor P) ou des colorations argentiques (Gomori-Grocott) peuvent augmenter la sensibilité dans les cas d'une faible densité fongique.

L'examen anatomopathologique des prélèvements biopsiques se fait après colorations spécifiques telles que Gomori-Grocott, PAS (periodic acid Schiff) ou HES (hématoxyline-éosine) [17]. Les caractéristiques morphologiques propres aux Mucorales (dans les produits pathologiques) sont les filaments mycéliens hyalins, larges (5 à $20 \mu \mathrm{m}$ ), à contours irréguliers, à paroi fine, rubanés, peu ou pas septés, et ayant souvent des ramifications à angles droits (Figure 2). La nature du mycélium (non septé) et des ramifications (angles droits) les distinguent de certains hyphomycètes du genre Aspergillus, Scedosporium ou Fusarium qui possèdent, eux, un mycélium septé avec des branchements notamment à angles aigus. II convient de noter que les coupes transversales des hyphes pourraient donner des images ressemblant à des levures ou des vacuoles. Ajoutons que dans certains cas (en particulier quand le traitement antifongique a débuté avant la biopsie) les caractéristiques morphologiques des hyphes des Mucorales peuvent être atypiques [21]. Ces champignons ont une forte capacité d'angio-invasion, ce qui explique leur dissémination rapide par voie hématogène. Nécrose, hémorragie et inflammation sont présentes dans les tissus infectés. Le diagnostic histologique de mucormycose permet ainsi d'instaurer un traitement ou une prise en charge adapté. L'examen anatomo-pathologique nécessite une véritable expertise et ne permet pas l'identification d'espèce [22, 23]. Des tests d'immunohistochimie, hybridation ou PCR in situ [24, 25] peuvent améliorer le diagnostic précoce de mucormycoses. Néanmoins, ces méthodes nécessitent une standardisation et une validation ultérieure.

\section{Mise en culture}

La mise en culture des prélèvements mycologiques sur des milieux sélectifs adaptés tels que le milieu de Sabouraud glucosé contenant des antibiotiques (chloramphénicol, gentamicine) est recommandée. L'utilisation des milieux additionnés de cycloheximide (actidione) n'est pas conseillée car la plupart des espèces de Mucorales sont inhibées, même à faibles concentrations, par cet inhibiteur de la synthèse protéique [26]. Le broyage des échantillons tissulaires d'ensemencer sur milieu en boîte de Petri que sur tube gélosé. Les températures d'incubation conseillées sont $25^{\circ} \mathrm{C}$ et $37^{\circ} \mathrm{C}$ pendant un minimum de 5 jours pour optimiser la croissance [27].

\section{Diagnostic moléculaire à partir}

des échantillons cliniques

Les approches moléculaires pour la détection des acides nucléiques dans des matériels cliniques peuvent permettre un diagnostic précoce. Plusieurs approches ont été décrites à partir des tissus à l'état frais, congelés ou fixés en paraffine. II n'existe pas actuellement de consensus sur les meilleurs paramètres techniques à utiliser. Dans la plupart des essais, les gènes de I'ADN ribosomique (18S, 28S, 5.8S) ainsi que les régions ITS1 (internal transcribed spacer) et ITS2 ont été choisies comme cibles. Diverses techniques de PCR classique mais également de PCR en temps réel ont été testées [17]. Malgré les avances prometteuses dans le diagnostic moléculaire des mucormycoses, les outils proposés nécessitent une standardisation, une optimisation et une validation approfondies avant d'envisager leur utilisation en routine dans les laboratoires de microbiologie.

\section{Cultures}

\section{Identification phénotypique}

L'identification morphologique d'une culture de mucorale requiert une solide expertise en matière de taxonomie. Cette identification est basée principalement sur la morphologie macro- et microscopique. D'autres critères sont pris en compte tels que les résultats des tests physiologiques ou la température maximale de croissance. La formation des zygospores (spores sexuées) n'a pas d'utilité pour l'identification en routine en raison du caractère hétérothallique de la plupart de ces champignons. La plupart de Mucorales sont thermotolérants et sont capables de pousser à des températures bien au-delà de $40^{\circ} \mathrm{C}$ et cela en 24 à 48 heures. Leur 


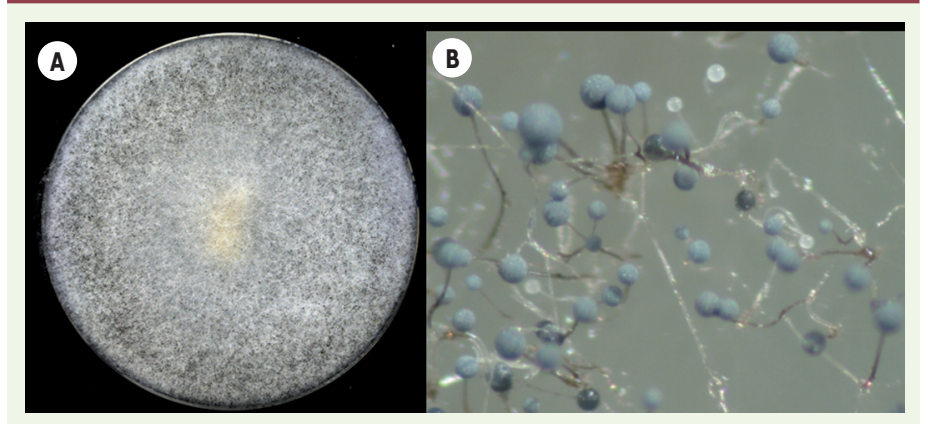

Figure 3. Aspect macroscopique d'une culture de Rhizopus sp. A. Sur milieu Malt $2 \%$ après 5 jours d'incubation à $30^{\circ} \mathrm{C}$. B. Observation de l'architecture fongique (sporocystophore, rhizoïdes, sporocystes) à la loupe binoculaire.

développement est rapide et extensif. Les milieux mycologiques à haute teneur en carbohydrates favorisent la croissance massive du mycélium, mais empêchent la sporulation des propagules asexuées requise pour l'identification. Les milieux malt $2 \%$ (MEA), pomme de terre-dextrose agar (PDA) sont recommandés pour une obtention optimale des fructifications. Des milieux pauvres en nutriments sont utilisés pour cibler des membres de Mucorales (Apophysomyces ou Saksenaea) qui ont des difficultés à sporuler dans les milieux habituels [28]. La température d'incubation utilisée pour la caractérisation phénotypique de ces champignons est de 27 à $30^{\circ} \mathrm{C}$, mais des tests de croissance à d'autres températures $\left(25^{\circ} \mathrm{C}, 37^{\circ} \mathrm{C}, 45^{\circ} \mathrm{C}\right.$ ) peuvent s'avérer utiles. Rappelons que les espèces de Mucorales les plus fréquemment rencontrées en pathologie humaine sont Rhizopus oryzae, Lichtheimia spp, Rhizomucor pusillus et Mucor spp [1, 2, 4].

\section{Examen macroscopique}

La plupart des colonies de Mucorales ont une texture floconneuse et des couleurs pouvant varier de blanc (Saksenaea) à jaune (Mucor), marron (Apophysomyces) ou gris (Lichtheimia, Rhizomucor). La hauteur du mycélium et le branchement du sporocystophore varient selon les différents genres des Mucorales. Ceci est observable à l'aide d'une loupe binoculaire (Figure 3).

\section{Examen microscopique}

L'examen microscopique de ces champignons repose principalement sur l'observation de certaines caractéristiques morphologiques telles que le branchement du sporocystophore, le type de sporocyste, la forme, la couleur, la présence ou l'absence d'apophyse et columelle et également la présence ou l'absence des rhizoïdes et des chlamydospores. Le diagramme d'identification illustré dans la Figure 4 permet d'établir une identification du genre en se basant sur la présence ou l'absence de ces éléments constitutifs. Les caractéristiques phénotypiques importantes des

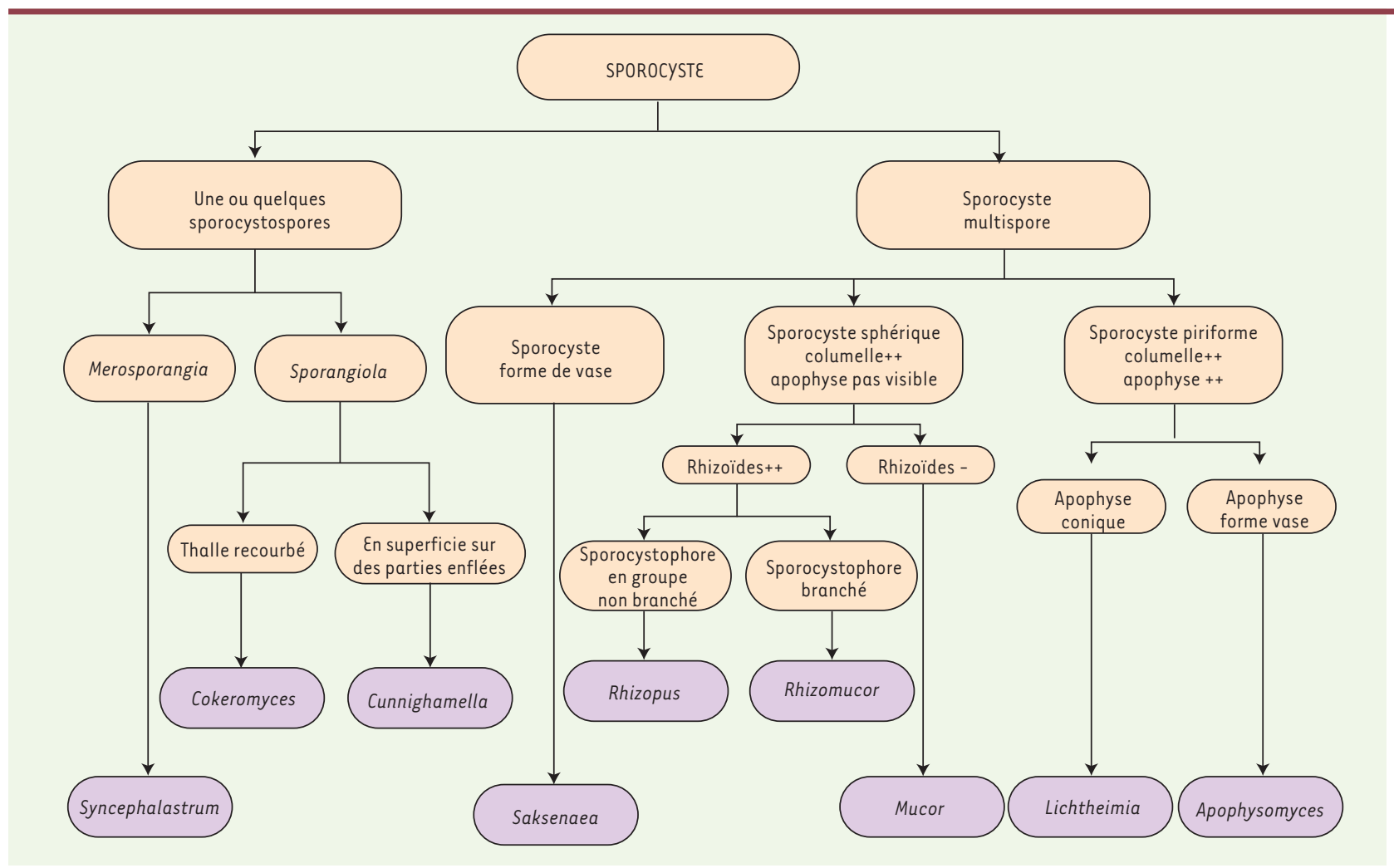

Figure 4. Diagramme décisionnel pour l'identification des différents genres des Mucorales. 


\begin{tabular}{|c|c|c|c|}
\hline Famille & Genre/espèce & Microscopie & Caractères phénotypiques importants \\
\hline \multirow[t]{4}{*}{ Mucoraceae } & $\begin{array}{l}\text { Rhizopus } \\
\text { oryzae }\end{array}$ & & $\begin{array}{l}\text { - Sporocystophore }=1 \mathrm{~cm} \text { de hauteur } \\
\text { - Rhizoïdes bien développés } \\
\text { - Sporocystospores striées } \\
\text { - Température maximale (T. max.) de croissance } 40^{\circ} \mathrm{C}\end{array}$ \\
\hline & $\begin{array}{l}\text { Rhizopus } \\
\text { microsporus }\end{array}$ & & $\begin{array}{l}\text { - Sporocystophore }=10 \mathrm{~mm} \text { de hauteur } \\
\text { - Rhizoïdes bien développés } \\
\text { - Sporocystospores striées } \\
\text { - T. max. de croissance } 50-52{ }^{\circ} \mathrm{C}\end{array}$ \\
\hline & $\begin{array}{l}\text { Rhizomucor } \\
\text { pusillus }\end{array}$ & & $\begin{array}{l}\text { - Sporocystophore branché en partie apical } \\
\text { - Sporocystes sans apophyse } \\
\text { - Rhizoïdes simples } \\
\text { - T. max. de croissance } 55^{\circ} \mathrm{C}\end{array}$ \\
\hline & $\begin{array}{l}\text { Mucor } \\
\text { circinelloides }\end{array}$ & & $\begin{array}{l}\text { - Sporocystophore pas branché ou irrégulièrement } \\
\text { branché } \\
\text { - Absence de rhizoïdes et stolons } \\
\text { - Oidia présent } \\
\text { - T. max. de croissance } 37^{\circ} \mathrm{C}\end{array}$ \\
\hline Lichthemiaceae & $\begin{array}{l}\text { Lichtheimia } \\
\text { corymbifera } \\
\text { ramosa }\end{array}$ & & $\begin{array}{l}\text { - Apophyse légèrement mélanisée } \\
\text { - Stolons et rhizoïdes présents } \\
\text { - Sporocystospores lisses } \\
\text { - T. max. de croissance entre } 45 \text { et } 52^{\circ} \mathrm{C}\end{array}$ \\
\hline Saksenaseae & Saksenaea spp & & $\begin{array}{l}\text { - Sporocystophore court } \\
\text { - Sporocystes en forme de vase } \\
\text { - Rhizoïdes pigmentés } \\
\text { - T. max. de croissance } 44{ }^{\circ} \mathrm{C}\end{array}$ \\
\hline Cunninghamellaceae & Cunnighamella spp & & $\begin{array}{l}\text { - Sporangiola portés sur des vésicules de la partie } \\
\text { apicale du sporocystophore } \\
\text { - Sporocystospores lisses ou finement échinulées } \\
\text { - T. max. de croissance } 46-50^{\circ} \mathrm{C}\end{array}$ \\
\hline
\end{tabular}

Tableau I. Principales espèces pathogènes des Mucorales (sous-phylum Mucoromycotina).

agents pathogènes les plus fréquemment rencontrés sont mentionnées dans le Tableau /. Divers ouvrages [9, 17, 29, 30] ainsi que certains sites web (www.cbs.knaw.nl, www.mycobank.org, www. mycology.adelaide.edu.au) sont également utiles pour identifier une souche de Mucorales au niveau de l'espèce.

\section{Identification moléculaire}

Depuis quelques années, différentes approches moléculaires ont été développées pour le diagnostic des infections à Mucorales [17]. Ce développement est stimulé par l'augmentation des cas d'infections fongiques invasives ou la meilleure prise en compte de leur importance, et par la lenteur diagnostique des méthodes classiques microbiologiques. Très souvent, l'identification morphologique n'aboutit pas à la détermination de genre ou d'espèce en raison de l'absence de fructifications de la souche ou la présence de caractères phénotypiques ne permettant pas de conclure. Les techniques moléculaires basées sur le séquençage visent donc à l'identification d'espèce grâce à l'amplification de régions d'intérêt taxonomique. Récemment, 
Schoch et al. ont proposé la région ITS de I'ADN ribosomique comme marqueur fongique principal dans la méthode de barcoding [31]. Dans le cas des Mucorales, le séquençage des ITS est suffisamment discriminant pour parvenir à une identification correcte tandis que le séquençage multigénique est utile pour des analyses phylogénétiques. Une identification correcte dépendra de la fiabilité des bases de données qui sont interrogées pour la recherche de similitude des séquences. Il existe dans les bases publiques des erreurs dues à des mauvaises séquences ou à une identification incorrecte. On peut noter que la technique de spectrométrie Matrix-assisted laser desorption ionization (MALDI)-time-of-flight (TOF) mass spectrometry (MS) a été mise au point récemment pour l'identification des champignons du genre Lichtheimia en routine, mais pas encore pour les autres genres.

\section{SUMMARY}

\section{Laboratory diagnosis of mucormycosis}

Mucormycosis are deep infections caused by ubiquitous filamentous fungi of the order of Mucorales. The disease occurs mostly in immunocompromised, diabetic or solid organ transplant recipients. There are currently no specific diagnostic guidelines for mucormycosis. The histological examination and culture of the clinical sample remain the most useful approaches for diagnosis. Furthermore, alternative methods to the fungal culture are yet to be standardized. Here we review the current microbiological approaches used for the diagnosis and identification of Mucorales. $\diamond$

\section{LIENS D'INTÉRÊT}

L'auteur déclare participer à des conférences pour l'entreprise Gilead Sciences.

\section{RÉFÉRENCES}

1. Lanternier F, Dannaoui $\varepsilon$, Morizot, G, et al. A global analysis of mucormycosis in France: the Retrozygo study (2005-2007). Clin Infect Dis 2012; 54 (suppl 1) : S35-43.

2. Roden MM, Zaoutis TE, Buchanan WL, et al. Epidemiology and outcome of zygomycosis: a review of 929 reported cases. Clin Infect Dis $2005 ; 41: 634-53$.

3. Singh N, Aguado JM, Bonatti H, et al. Zygomycosis in solid organ transplant recipients: a prospective, matched case-control study to assess risks for disease and outcome. J Infect Dis $2009 ; 200: 1002-11$.

4. Skiada A, Pagano L, Groll A, et al. Zygomycosis in Europe: analysis of 230 cases accrued by the registry of the European Confederation of Medical Mycology (ECMM) Working Group on Zygomycosis between 2005 and 2007. Clin Microbiol Infect 2011; 17: 1859-67.

5. Lanternier F, Sun Hy, Ribaud P, et al. Mucormycosis in organ and stem cell transplant recipients. Clin Infect Dis $2012 ; 54: 1629-36$.

6. Adam RD, Hunter G, DiTomasso J, Comerci G Jr. Mucormycosis: emerging prominence of cutaneous infections. Clin Infect Dis $1994 ; 19: 67-76$.

7. Kontoyiannis DP, Marr KA, Park BJ, et al. Prospective surveillance for invasive fungal infections in hematopoietic stem cell transplant recipients, 2001-2006: overview of the Transplant-Associated Infection Surveillance Network (Transnet) Database. Clin Infect Dis 2010 ; $50: 1091-100$.

8. Bitar D, Van Cauteren D, Lanternier F, et al. Increasing incidence of zygomycosis (mucormycosis), France, 1997-2006. Emerg Infect Dis 2009; 15 : 1395-401.

9. Ribes JA, Vanover-Sams CL, Baker DJ. Zygomycetes in human disease. Clin Microbiol Rev 2000 ; 13 : 236-301.

10. Rammaert B, Lanternier F, Zahar JR, et al. Healthcare-associated mucormycosis. Clin Infect Dis $2012 ; 54$ (suppl 1) : S44-54.

11. Voigt $K$, Cigelnik $\varepsilon$, O'Donnell K. Phylogeny and PCR identification of clinically important Zygomycetes based on nuclear ribosomal-DNA sequence data. J Clin Microbiol 1999 ; 37 : 3957-64.
12. Voigt $K$, Wostemeyer J. Phylogeny and origin of 82 zygomycetes from all 54 genera of the Mucorales and Mortierellales based on combined analysis of actin and translation elongation factor EF-lalpha genes. Gene $2001 ; 270$ : 113-20.

13. O’Donnell K, Lutzoni F, Ward TJ, Benny GL. Evolutionary relationships among mucoralean fungi (Zygomycota): evidence for family polyphyly on a large scale. Mycologia $2001 ; 93: 286-96$.

14. Hibbett DS, Binder M, Bischoff JF, et al. A higher-level phylogenetic classification of the Fungi. Mycol Res 2007 ; 111 : 509-547.

15. Ruping MJ, Heinz WJ, Kindo AJ, et al. Forty-one recent cases of invasive zygomycosis from a global clinical registry. J Antimicrob Chemother 2010 ; $65: 296-302$.

16. Alvarez $\varepsilon$, Sutton DA, Cano J, et al. Spectrum of zygomycete species identified in clinically significant specimens in the United States. J Clin Microbiol $2009 ; 47: 1650-6$.

17. Garcia-Hermoso D, Dannaoui $\varepsilon$, Lortholary 0, Dromer F. Agents of systemic and subcutaneous mucormycosis and entomophthoromycosis. In : Manual of clinical microbiology. Washington DC : American Society for Microbiology, 2011.

18. Haurany N, Kontoyiannis DP, Buchanan M, et al. Interobserver reproducibility and concordance between culture and histopathology in invasive mold infections: a multicenter study. In : $4^{\text {th }}$ Advances against Aspergillosis. Rome, Italy, 2010 (abstract 78).

19. De Pauw B, Walsh TJ, Donnelly JP, et al. Revised definitions of invasive fungal disease from the European Organization for Research and Treatment of Cancer/Invasive Fungal Infections Cooperative Group and the National Institute of Allergy and Infectious Diseases Mycoses Study Group (EORTC/ MSG) Consensus Group. Clin Infect Dis 2008 ; 46 : 1813-21.

20. Ayats J, Martin-Mazuelos $\varepsilon$, Peman J, et al. Spanish Society of Clinical Microbiology and Infectious Diseases (SEIMC) guidelines for the diagnosis of invasive fungal infections. 2010 update. Enferm Infect Microbiol Clin 2011 ; $29: 39$ e31-15.

21. Walsh TJ, Gamaletsou MN, McGinnis MR, et al. Early clinical and laboratory diagnosis of invasive pulmonary, extrapulmonary, and disseminated mucormycosis (zygomycosis). Clin Infect Dis 2012; 54 (suppl 1) : S55-60.

22. Frater JL, Hall GS, Procop GW. Histologic features of zygomycosis: emphasis on perineural invasion and fungal morphology. Arch Pathol Lab Med 2001 ; $125: 375-8$.

23. Hammond SP, Bialek R, Milner DA, et al. Molecular methods to improve diagnosis and identification of mucormycosis. J Clin Microbiol $2011 ; 49$ : 2151-3.

24. Hayden RT, Qian X, Procop GW, et al. In situ hybridization for the identification of filamentous fungi in tissue section. Diagn Mol Pathol 2002 ; $11: 119-26$.

25. Montone KT, LiVolsi VA, Lanza DC, et al. In situ hybridization for specific fungal organisms in acute invasive fungal rhinosinusitis. Am J Clin Pathol $2011 ; 135: 190-9$.

26. Schwarz P, Lortholary 0, Dromer F, Dannaoui $\varepsilon$. Carbon assimilation profiles as a tool for identification of zygomycetes. J Clin Microbiol 2007 ; 45 : 1433-9.

27. Kontoyiannis DP, Chamilos G, Hassan SA, et al. Increased culture recovery of Zygomycetes under physiologic temperature conditions. Am J Clin Pathol $2007 ; 127: 208-12$.

28. Alvarez $\varepsilon$, Garcia-Hermoso D, Sutton DA, et al. Molecular phylogeny and proposal of two new species of the emerging pathogenic fungus Saksenaea. J Clin Microbiol $2010 ; 48: 4410-6$.

29. De Hoog GS, Guarro J, Gené J, Figueras MJ. Atlas of clinical fungi $C D-R O M$. A pilot CD-ROM version of the $3^{\text {rd }}$ ed. Utrecht, The Netherlands : Centraalbureau voor Schimmelcultures, 2009.

30. Ellis D, Davis S, Alexiou H, et al. Descriptions of medical fungi. Adelaide, Australia, 2007.

31. Schoch CL, Seifert KA, Huhndorf S, et al. Nuclear ribosomal internal transcribed spacer (ITS) region as a universal DNA barcode marker for Fungi. Proc Natl Acad Sci USA 2012 ; 109 : 6241-6.

\section{TIRÉS À PART}

D. Garcia-Hermoso 